\title{
Fatores Associados à Realização de Cesárea em Primíparas com uma Cesárea Anterior
}

\author{
Factors Associated with Cesarean Section in Primipara Women with One Previous \\ Cesarean Section
}

\begin{abstract}
José Guilherme Cecatti, Carla Betina Andreucci, Pilar Souza Cacheira, Helaine Maria Besteti Pires,
\end{abstract} João Luiz Pinto e Silva, Márcia Maria Auxiliadora de Aquino

\section{RESUMO}

Objetivo: avaliar a via de parto em um grupo de gestantes primiparas de baixa renda com uma cesárea anterior e os fatores associados à repetição da cesárea no segundo parto.

Pacientes e Métodos: realizou-se um estudo caso-controle com 356 gestantes atendidas de janeiro de 1993 a janeiro de 1996 na Maternidade do CAISM/UNICAMP. Constituiram os casos as 153 gestantes que tiveram o segundo parto por cesárea, e os controles, as 203 que tiveram o segundo parto vaginal. Para a análise utilizaram-se médias, desvio padrão, teste $\mathrm{t}$ de Student, teste de Mann-Whitney, $\chi^{2}$ e "odds ratio" (OR) e IC 95\% para cada possivel fator associado à realização de cesárea no segundo parto.

Resultados: a via do segundo parto foi vaginal em $57 \%$ das vezes. Dentre as diversas variáveis estudadas, as que mostraram estar significativamente associadas à realização de cesárea no segundo parto foram: maior idade materna (para mulheres com 35 anos ou mais, OR =16,4), antecedente de abortamento $(O R=2,09)$, indução do trabalho de parto $(O R=3,83)$, rotura prematura de membranas $(O R=2,83)$, a não-realização de analgesia durante o periodo de dilatação $(O R=5,3)$, o diagnóstico de algum sinal de vitalidade fetal alterada $(O R=2,7)$ e a ocorrência do parto à tarde $(O R=1,92)$.

Conclusões: os resultados indicam que os fatores associados à repetição de cesárea em mulheres com uma cicatriz de cesárea nesta população são predominantemente médicos, mas há a possibilidade de se proporem intervenções dirigidas a diminuir o índice de repetição de cesáreas.

PALAVRAS-CHAVE: Cesariana. Analgesia de parto. Trabalho de parto.

\section{Introdução}

Historicamente a cesárea surgiu como alternativa para situações extremas, como recurso para salvaguardar a vida do concepto ou da mãe $e^{1}$. Até o final do século XIX, as estatísticas mundiais mostravam sobrevida materna à operação cesariana que não ultrapassava $50 \%{ }^{2}$. A partir do início da segunda metade deste século, as técnicas ci-

Departamento de Tocoginecologia, Centro de Atenção Integral à Saúde da Mulher, Universidade Estadual de Campinas

Correspondência:

JG Cecatti

Rua Alexander Fleming, 101

13081-970 - Campinas - SP

Fax: (19)788-9304 rúrgicas e de retaguarda evoluíram para oferecer significativa melhoria dos resultados obstétricos maternos e perinatais, o que causou um rápido aumento no número de indicações de parto abdominal.

Durante praticamente toda a década de setenta até quase metade da década de oitenta, supunha-se que a cesariana melhorava o prognóstico perinatal, o que serviu como justificativa para o aumento do número de cesáreas neste período. Hoje já se sabe que os principais determinantes da diminuição da morbimortalidade perinatal em décadas anteriores foram os avanços no tipo, qualidade e disponibilidade dos serviços médicos em geral e dos obstétricos e neonatais em particular, com a melhoria global na atenção à saúde mater- 
na, sem relação com a via de parto ${ }^{3}$.

A resolução do parto mediante uma cesariana é atualmente uma ocorrência comum em quase todos os países do mundo, sobretudo no Brasil, onde o fato ocorre em niveis epidêmicos em algumas regiões. No Rio de Janeiro e em São Paulo, as taxas de cesárea chegam a ultrapassar $50 \%$ do total de partos ${ }^{3}$.

Vários fatores têm sido relacionados à indicação da via de resolução do parto: condição sócioeconômica, indicação médica, opção da parturiente ou do obstetra, condições institucionais e, entre outros, o antecedente de cesárea. Na atualidade podem-se observar as conseqüências resultantes da realização indiscriminada de cesáreas pelo aumento de complicações obstétricas como placenta prévia, infecções, hemorragias e os vários graus de acretismo placentário ${ }^{3,4}$.

$\mathrm{Na}$ atualidade, as indicações para uma cesárea se ampliaram muito, na maior parte das vezes sem justificativa obstétrica adequada. Em 1984 , as cesáreas de repetição respondiam por 36\% das indicações de cesáreas ${ }^{4}$. Entre 1991 e 1992, 35\% das cesáreas realizadas nos Estados Unidos tiveram como principal indicação o antecedente de cesárea ${ }^{5}$.

A justificativa para a realização de uma cesárea em paciente com cesárea anterior é bastante antiga, porém não apresenta substrato obstétrico na atualidade. Vários trabalhos mostram boas chances de sucesso e praticamente ausência de riscos maternos e fetais com a realização de parto vaginal nesta condição ${ }^{2,6,7}$.

A realização da prova de trabalho de parto em gestantes com cesárea anterior é procedimento seguro, com boas chances de sucesso e uma das medidas possiveis para se reduzirem as taxas de repetição de cesárea e os custos, tanto em termos de morbimortalidade materna e perinatal quanto hospitalares, associados à realização de partos abdominais.

Além dos fatores clínicos, outros fatores encontram-se associados à repetição do parto abdominal em mulheres com uma cesárea anterior (que é na atualidade uma das principais indicações de cesárea). Um estudo americano mostrou que algumas características da instituição associam-se a um maior risco de uma segunda cesárea. Mostrou que o risco é significativamente maior nas instituições não-universitárias, privadas e com baixo volume obstétrico ${ }^{8}$. Discute-se também a ausência de enfermeiras obstétricas em algumas instituições como fator associado ao incremento de cesárea ${ }^{6}$.

Fatores inerentes ao médico, como conveniência e insegurança devida à treinamento obstétrico insatisfatório, também se associam ao maior risco de outra cesárea ${ }^{2}$. Estudo realizado nos Estados Unidos constatou que o médico tem uma influência importante sobre a incidência de cesáreas, perdendo apenas para a nuliparidade ${ }^{9}$.

Além dos fatores relacionados à instituição e ao médico, existem outros fatores não-clínicos associados a um risco maior de outra cesárea. Dizem respeito à mulher e aos seus aspectos culturais. Quanto à mulher, sua preferência por cesárea relaciona-se ao medo da dor e falta de preparo no pré-natal para o parto vaginal. Quanto aos fatores culturais, os conceitos populares de que o parto vaginal leva à perda acentuada da função sexual e de que o parto vaginal é mais arriscado para o feto do que uma cesárea associam-se também ao maior risco de um parto abdominal ${ }^{2}$.

O objetivo deste estudo foi avaliar a via de parto em gestantes primíparas com uma cesárea anterior e os fatores associados à repetição da cesárea no segundo parto em uma população de baixa renda atendida em hospital público universitário. O conhecimento desses fatores poderá auxiliar na proposta de alternativas que ajudem a reverter a situação atual, colaborando na redução das taxas de cesárea.

\section{Pacientes e Métodos}

Trata-se de um estudo do tipo caso-controle realizado na Maternidade do Centro de Assistência Integral à Saúde da Mulher (CAISM), da Faculdade de Ciências Médicas da Universidade Estadual de Campinas (FCM/UNICAMP), em Campinas, SP. A análise, com dados retrospectivamente coletados, abrangeu o período de janeiro de 1993 a janeiro de 1996, sendo os dados coletados a partir de ficha obstétrica pré-codificada, utilizando um formulário padronizado. Constituíram os sujeitos 356 gestantes primíparas a termo (idade gestacional $\geq 37$ semanas), com uma cesárea anterior, admitidas para parto na instituição. Os casos foram 153 gestantes com segundo parto por cesárea, e os controles, 203 gestantes com segundo parto por via vaginal. Foram analisadas a indicação de cesárea neste segundo parto, o índice de Apgar ao $1^{\circ}$ e $5^{\circ}$ minutos e, como variáveis de controle, o número de consultas de pré-natal, mês de seu início, idade gestacional por ocasião do parto, tempo de rotura de membranas e tempo desde a admissão ao parto. Também foram avaliadas as variáveis independentes potencialmente associadas à decisão pela via do segundo parto: idade materna, situação marital, escolaridade, local do pré-natal, antecedentes mórbidos, uso de ocitocina, dia da semana, antecedente de abor- 
tos, analgesia de parto, indução do trabalho de parto, rotura prematura de membranas, alteração da vitalidade fetal e horário do parto. Para a análise estatística utilizaram-se média, DP e teste $t$ de Student e Mann-Whitney para variáveis numéricas, $\chi^{2}$ para variáveis categóricas e estimativa de risco pelo "odds ratio" (OR) e IC 95\% para fatores possivelmente associados à realização de cesárea no segundo parto.

\section{Resultados}

Repetiu-se o parto cirúrgico em $43 \%$ das gestantes, tendo $57 \%$ evoluído para parto vaginal. Este foi, portanto, o índice de parto vaginal após cesárea (PVAC) nesta população, independente da indicação da primeira cesárea. As indicações mais fre-

Tabela 1 - Distribuição das mulheres que tiveram o segundo parto por cesárea, segundo a indicação.

\begin{tabular}{lcr}
\hline Indicação & $\mathbf{n}$ & \multicolumn{1}{c}{$\%$} \\
\hline Somatória (inclui cesárea anterior) & 43 & 28,1 \\
Sofrimento fetal & 39 & 25,5 \\
Desproporção céfalo-pélvica & 13 & 8,5 \\
Outras distócias & 13 & 8,5 \\
Hipertensão arterial & 3 & 2,0 \\
Placenta prévia & 2 & 1,3 \\
Acidente de cordão & 2 & 1,3 \\
Outros & 38 & 25,0 \\
Total & 153 & 100,0 \\
\hline
\end{tabular}

qüentes para cesárea no segundo parto foram: somatória de várias causas, sofrimento fetal, desproporção céfalo-pélvica (DCP), outras distocias, hipertensão arterial, placenta prévia, acidente de cordão e outras (Tabela 1). A média de idade gestacional foi semelhante nos dois grupos $(39,4$ semanas), da mesma forma que o número de consultas e a época de início do pré-natal. O tempo desde a rotura de membranas até o parto e o tempo da admissão até o parto não apresentaram diferenças significativas em nenhum dos dois grupos (Tabela 2).

A estimativa de risco para o segundo parto por cesárea não foi significativa para algumas variáveis demográficas e da gestação: situação marital estável, baixa escolaridade (até 4 anos), pré-natal fora da instituição, antecedentes maternos mórbidos, partos ocorridos durante o fim-desemana e o uso de ocitocina (Tabela 3).

Tabela 2 - Valores médios de algumas variáveis da gravidez e da assistência de pré-natal segundo a via do segundo parto.

\begin{tabular}{|c|c|c|c|}
\hline Variáveis & $\begin{array}{c}\text { Cesárea } \\
\text { Média } \pm \text { DP }\end{array}$ & $\begin{array}{c}\text { Vaginal } \\
\text { Média } \pm \text { DP }\end{array}$ & p \\
\hline $\mathrm{N}^{0}$ de consultas de pré-natal & $7,3 \pm 3,3$ & $6,8 \pm 2,9$ & $\mathrm{NS}^{*}$ \\
\hline Mês de início de pré-natal & $4,2 \pm 1,9$ & $4,3 \pm 1,9$ & $\mathrm{NS}^{*}$ \\
\hline Idade gestacional ao nascimento & $39,4 \pm 1,9$ & $39,4 \pm 1,8$ & $\mathrm{NS}^{*}$ \\
\hline Tempo rotura membranas-parto (h) & $4,3 \pm 9,5$ & $5,1 \pm 9,7$ & $\mathrm{NS}^{*}$ \\
\hline Tempo admissão-parto (h) & $10,5 \pm 13,4$ & $8,2 \pm 9,2$ & $N S^{* *}$ \\
\hline
\end{tabular}

Tabela 3 - Distribuição percentual de algumas variáveis demográficas e da gestação com estimativa de risco para o segundo parto por cesárea.

\begin{tabular}{lcccc}
\hline Variável & Cesárea & Vaginal & OR & IC 95\% \\
\hline Situação marital estável & 91,3 & 89,1 & 1,29 & $0,60-2,82^{*}$ \\
Baixa escolaridade (até 4 anos) & 34,5 & 26,8 & 1,44 & $0,82-2,52^{*}$ \\
Pré-natal fora da instituição & 58,6 & 59,5 & 0,88 & $0,55-1,40^{*}$ \\
Antecedente mórbido (+) & 38,6 & 37,9 & 1,03 & $0,65-1,62^{*}$ \\
Parto no fim-de-semana & 25,5 & 25,6 & 0,99 & $0,60-1,65^{*}$ \\
Uso de ocitocina & 26,9 & 27,6 & 0,96 & $0,58-1,60^{*}$ \\
\hline
\end{tabular}

* Não-significante.

Observou-se um aumento progressivo e significativo no risco do segundo parto por cesárea com o avançar da idade materna. Assim, as mulheres com idade entre 30 a 35 anos tiveram um risco 5,1 vezes maior de cesárea que as com idade abaixo de 20 anos e para as com 35 anos ou mais, este risco foi 16,4 vezes maior. Da mesma forma, a idade média foi maior no grupo de mulheres com cesárea no segundo parto (Tabela 4). Também foi cerca de duas vezes maior o risco do segundo parto por cesárea com o antecedente de abortos anteriores (Tabela 5). 
Tabela 4 - Distribuição percentual da idade materna, com estimativa de risco para 0 segundo parto por cesárea.

\begin{tabular}{lcccc}
\hline Idade & Cesárea & Vaginal & OR & IC 95\% \\
\hline $14-19$ & 6,6 & 20,3 & 1,00 & Ref \\
$20-24$ & 29,1 & 37,1 & 2,41 & $1,03-5,70$ \\
$25-29$ & 34,4 & 30,2 & 3,50 & $1,51-8,28$ \\
$30-34$ & 16,6 & 9,9 & 5,13 & $1,90-14,15$ \\
$35+$ & 13,2 & 2,5 & 16,40 & $4,38-67,18$ \\
Total $(n) *$ & 151 & 202 & - & - \\
Média \pm DP & $27,4 \pm 6,1$ & $23,9 \pm 4,9$ & $T=5,72$ & $p<0,0001$ \\
\hline$\chi_{\text {trend }}^{2}=29,02$ & p<0,00001 & & & \\
${ }^{*}$ Faltam informações para 2 casos e 1 controle & &
\end{tabular}

Tabela 5 - Distribuição percentual de algumas variáveis relacionadas ao parto com estimativa de risco para o segundo parto por cesárea.

\begin{tabular}{lcccc}
\hline Variáveis & Cesárea & Vaginal & OR & IC 95\% \\
\hline $\begin{array}{c}\text { Antecedente de } \\
\text { aborto }\end{array}$ & 28,1 & 15,7 & 2,09 & $1,21-3,62$ \\
$\begin{array}{c}\text { Não-realização de } \\
\text { analgesia }\end{array}$ & 87,2 & 56,3 & 5,30 & $2,94-9,64$ \\
$\begin{array}{c}\text { Trabalho de parto } \\
\text { induzido }\end{array}$ & 19,6 & 6,0 & 3,83 & $1,72-8,64$ \\
$\begin{array}{c}\text { Rotura prematura } \\
\text { de membranas }\end{array}$ & 30,3 & 13,3 & 2,83 & $1,42-5,62$ \\
$\begin{array}{c}\text { Alterações da } \\
\text { vitalidade fetal }\end{array}$ & 58,2 & 33,9 & 2,70 & $1,71-4,27$ \\
$\begin{array}{c}\text { Parto à tarde } \\
(13-18 \mathrm{~h})\end{array}$ & 41,2 & 26,7 & 1,92 & $1,20-3,08$ \\
\hline
\end{tabular}

Observou-se ainda um risco cinco vezes maior de parto cesárea entre as gestantes não submetidas à analgesia de parto, quase quatro vezes maior com indução do trabalho de parto, quase três vezes maior com rotura prematura de membranas, 2,7 vezes maior com alterações da vitalidade fetal e quase duas vezes maior com partos ocorridos no período da tarde, entre as 13 e 18 horas (Tabela 5). A porcentagem de baixo índice de Apgar dos recém-nascidos no primeiro minuto de vida foi maior no grupo da cesárea $(18,3 \%$ e $8,4 \%, p<0,01)$, mas esta diferença não foi observada no quinto minuto de vida $(1,3 \%$ e $0,5 \%, p=0,39)$.

\section{Discussão}

Este estudo mostrou que, nesta população brasileira de gestantes primiparas de baixa renda e com uma cesárea anterior, os fatores mais importantes que estiveram associados à repetição do parto abdominal foram: idade materna maior do que 19 anos, o antecedente de abortamento, a presença de rotura prematura de membranas, diagnóstico de alguma alteração da vitalidade fetal e algumas variáveis relacionadas à prova de trabalho de parto.
Nesta investigação houve uma tendência estatisticamente significativa de maior risco de ocorrência de um segundo parto cesárea nas mulheres com idade superior a 19 anos e naquelas com antecedente de abortamento. Outro estudo brasileiro realizado em 1998 também encontrou maior risco para outra cesárea nas mulheres com idade a partir de $35 \operatorname{anos}^{10}$, e esta é uma tendência conhecida, embora não existam justificativas biológicas claras para explicá-la. Já para o antecedente de abortamento, também associado à decisão pela cesárea no segundo parto, não existe explicação convincente. Uma hipótese exploratória seria talvez a cesárea como conseqüência à maior valorização dada à gravidez, tanto pela mulher quanto pelo médico, no caso de perdas anteriores.

O risco para ocorrência de uma segunda cesárea nas mulheres do estudo foi quase três vezes maior quando havia rotura prematura de membranas; risco semelhante foi também estimado na presença de alterações da vitalidade fetal. Nesta última situação, deve-se tomar cuidado para que não haja interpretação equivocada dos sinais de vitalidade fetal, devido ao momento ou tempo de realização do exame inadequados e/ou técnica imprópria que levem a um precipitado e errôneo diagnóstico de sofrimento fetal agudo, provavelmente muito freqüente no Brasil ${ }^{11}$.

Este estudo também mostrou que quando a realização do parto ocorreu entre as 13 e as 18 horas houve um risco significativo de ocorrência de cesárea, sendo quase duas vezes maior em relação ao parto vaginal. Isto reitera o papel do médico na decisão de uma cesárea, haja vista a conhecida tendência de não se "passar o plantão" com casos não-resolvidos, que pode estar possivelmente envolvida na decisão do parto abdominal, pelo menos em alguma proporção deles.

Os resultados deste estudo mostraram ainda que variáveis relacionadas à prova de trabalho de parto estiveram fortemente associadas ao risco de uma segunda cesárea. A não-realização de analgesia durante o trabalho de parto associou-se a um risco cinco vezes maior de ocorrência de parto cesárea. Quando houve indução do parto, este risco foi de cerca de quatro vezes. Isto mostra a necessidade de protocolos específicos para prova de trabalho em mulheres com cicatriz uterina prévia que considerem o envolvimento do médico e da mulher na decisão, monitorização do parto, ocitocina em baixas doses, analgesia e vigilância contínua do obstetra, fatores já discutidos por vários outros autores ${ }^{12}$. Pickhardt et al. ${ }^{13}$, estudando parto vaginal após cesárea, concluem sobre a necessidade do encorajamento de uma prova de trabalho de parto em todas as mulheres com uma cesárea prévia, ao menos que haja uma forte indicação médica para não realizá-la. Diversos outros autores têm mostrado que esta prova de tra- 
balho permite atingir cifras em torno de pelo menos $50 \%$ de partos por via vaginal ${ }^{11}$.

Estudo realizado por Kline e Arias ${ }^{14}$ mostrou que indicações médicas e o desejo da mulher respondem por $77,4 \%$ das decisões de uma segunda cesárea, enfatizando que o medo da dor e as "inconveniências" de um trabalho de parto associados à lembrança do êxito da primeira cesárea são motivos fortes para a opção por um segundo parto abdominal, apontando para a necessidade de melhor informação sobre os riscos associados a este procedimento.

Este estudo permitiu ainda concluir que, se além dos fatores médicos e obstétricos, vários outros estão associados à realização de cesárea no segundo parto de uma gestante com cesárea anterior, seria razoável propor medidas para favorecer o PVAC, atuando sobre alguns fatores, como permitir a prova de trabalho de parto, realizar analgesia, evitar indução com ocitocina exclusiva e adotar melhores critérios para o diagnóstico de sofrimento fetal e DCP, dentre outros. Isto acabaria tendo um forte impacto na diminuição da incidência de cesárea e, portanto, na incidência das complicações a ela associadas e nos custos hospitalares de sua realização.

Além disso, mostrou ainda que, numa população de baixa renda atendida em hospital público universitário, os fatores não-médicos são menos importantes na decisão por uma segunda cesárea, ao contrário do que foi encontrado estudando-se uma população geral no mesmo município ${ }^{15}$.

\section{SUMMARY}

Purpose: to evaluate the route of delivery in a group of lowincome primipara pregnant women with a previous cesarean section, and the factors associated with the repetition of the cesarean section on the second delivery.

Patients and Methods: it was a case-control study including 356 women who were assisted at the Maternity of CAISM/ UNICAMP during the period between January 1993 and January 1996. The cases were 153 women whose second delivery was through a cesarean section and the controls were 203 women whose second delivery was vaginal. For analysis, means, standard deviation, Student's t-test, MannWhitney test, $\chi^{2}$ test and odds ratio (OR) with 95\% CI for each factor possibly associated with cesarean section on the second delivery were used.

Results: the route of the second delivery was vaginal for $57 \%$ of the women. Among the several variables studied, those which showed to be significantly associated with a cesarean section on the second delivery were: higher maternal age (for women over 35 years, $O R=16.4$ ), previous abortions $(O R=2.09)$, induced labor $(O R=3,83)$, premature rupture of membranes $(O R=2.83)$, not having an epidural analgesia performed during labor $(O R=5.3)$, the finding of some alteration in fetal well-being $(O R=2.7)$ and the delivery occurring during the afternoon $(O R=1.92)$.
Conclusions: these results indicate that the factors associated with the repetition of cesarean section in women with a previous scar of cesarean section in this population are predominantly medical; however, there is still the possibility of proposing interventions directed to decreasing the rates of repeated cesarean sections.

KEY WORDS: Cesarean section. Vaginal birth. Risk factors.

\section{Referências}

1. Katz VL, Cefalo RC. History and evolution of cesarean delivery. In: Phelan JP, Clark SL, editores. Cesarean Delivery. $1^{\text {st }}$ ed. New York: Elsevier Science Publishing; 1988. p.1-18.

2. Francome C, Savage W, Churchill H, Lewison H. Caesarean birth in Britain. $1^{\text {st }}$ ed. London: Middlesex University Press; 1993.

3. Faúndes A, Cecatti JG. A operação cesárea no Brasil: incidência, tendências, causas, conseqüências e propostas de ação. In: Giffin KM, editor Mulher e Saúde. Rio de Janeiro: Fundação Oswaldo Cruz; 1991. p.150-73.

4. Shiono PH, McNellis D, Rhoads GG. Reasons for the rising cesarean delivery rates: 1978-1984. Obstet Gynecol 1987; 69:696-700.

5. Paul RH, Miller DA. Cesarean birth: how to reduce the rate. Am J Obstet Gynecol 1995; 172:1903-11.

6. O’Sullivan MJ, Fumia F, Holsinger K, McLeod AG. Vaginal delivery after caesarean section. Clin Perinatol 1981; 8:131-43

7. Flamm BL, Goings JR, Liu Y, Wolde-Tsadik G. Elective repeat cesarean delivery versus trial of labor: a prospective multicenter study. Obstet Gynecol 1994; 83:927-32.

8. Stafford RS. The impact of nonclinical factors on repeat cesarean section. JAMA 1991; 265: 59-63.

9. Goyert GL, Bottoms SF, Treadwell MC, Nehra PC. The physician factor in cesarean birth rates. N Engl J Med 1989; 320:706-9.

10.Santos LC, Amorim MMR, Porto AMF, Azevedo EB, Mesquita CC. Fatores prognósticos para o parto transvaginal em pacientes com cesárea anterior. Rev Bras Ginecol Obstet 1998; 20:342-9.

11.Faúndes A, Pinto e Silva JL. O equilíbrio entre o parto vaginal e a cesárea na assistência obstétrica atual. Femina 1998; 26:669-73.

12.Morais EN, Mauad Filho F, Gallareta FMP, Spara P. Parto vaginal após cesárea prévia - Uma análise critica. Ginecol Obstet Atual 1998; 7:20-32.

13.Pickhardt MG, Martin JN, Meydrech EF, Blake PG, Martin RW, Perry KG, et al. Vaginal birth after cesarean delivery. Are those useful and valid predictors of success or failure? Am J Obstet Gynecol 1992; 166:1811-19.

14.Kline J, Arias F. Analysis of factors determining the selection of repeated cesarean section or trial of labor in patients with histories of prior cesarean delivery. $\mathrm{J}$ Reprod Med 1993; 38:289-92.

15.Pires HMB, Cecatti JG, Faúndes A. Fatores associados à prova de trabalho de parto em primiparas com uma cesárea anterior. Rev Saúde Públ 1999; 33:342-8. 